\title{
Understanding what it means to be a health practitioner: biographies of care and concern
}

\author{
Wendy McMillan
}

I am not a health practitioner, although I work in a Health Sciences Faculty. As the faculty's 'Education Advisor', I am intimately involved in the education of health practitioners. I mentor students to learn more effectively and work with teachers to help prepare clinically competent, caring professionals. Perhaps because I am not medically trained, I evaluate graduates through the eyes of a patient-consumer. I am obviously concerned with clinical competence. But I also want to know, 'will this graduate professional understand me as a person, viewing my health care as integral to who I am as a human being, collaborating with me to ensure my best quality of life?' Growing research informs on how to teach for technical competence, ensuring that graduates display the knowledge and skills prerequisite for accurate diagnosis and appropriate treatment. My concern is for nurturing 'what it means to be human': i.e. helping students to develop moral wisdom, ${ }^{1}$ and to improve their empathy for patients' experiences. ${ }^{2}$ This might, I argue, be achieved through engaging students in fictional and biographical accounts of the lives of health practitioners and patients.

Medicine provides knowledge about the health of a person. Literature provides insight into the meaning of an individual's experience. ${ }^{3}$ Stories offer students opportunities to live in the 'skin' of another, and to comprehend a patient's illness and treatment from the patient's point of view. ${ }^{4}$ Stories engage readers more fully than do clinical descriptions because they mobilise the imagination. ${ }^{5}$ Stories clarify the roles and expectations of health practitioners. ${ }^{4}$ Stories frame events and emotions in ways that encourage student- readers to examine critically all that a health practitioner is called upon to do. ${ }^{4}$ They provide students with language and tools for thinking about and managing, not only their patients, but also themselves and their own emotions. ${ }^{6}$ They alert students to the uncertainty and ambiguity intrinsic to health care practice. ${ }^{6}$ By engaging with literature, students may have their existing beliefs and assumptions challenged. They may or may not agree with the actions of protagonists, but they are unable to ignore the meaning and significance of these actions. ${ }^{7}$ Health care narratives thus signal to students that health care practice is more than technical and scientific competence. It encompasses acts of interpretation and contemplation. 4 


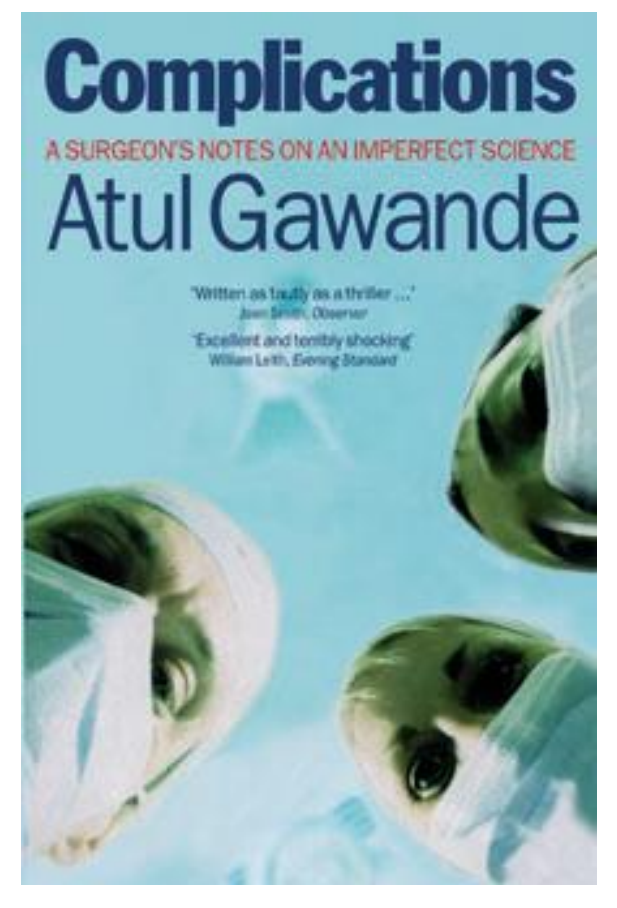

I have selected three books that exemplify such learning opportunities. Atul Gawande's autobiographical account, Complications, ${ }^{8}$ is presented in three sections. The first examines the fallibility of doctors, illustrating the decisions that doctors have to make when information is inadequate, when the available science is ambiguous, and when the attending doctor's knowledge and abilities are, of necessity, imperfect. Gawande explores the challenge to accurate diagnosis presented by the 'human-ness' of the health practitioner. Humans are inconsistent, influenced by suggestion, the order in which things are perceived, recent experiences, distractions and the way in which information is framed. ${ }^{9}$ He unpacks the tension between the patient's right to the best care possible and the need for future health practitioners to practice on living patients. The second section illustrates the 'mysteries and unknowns of medicine' (such as chronic pain with no identifiable physical abnormality), and explores how health practitioners perceive and struggle with what to do about these 'ever- present reminder(s) of failure'. The final section engages with health care uncertainty itself, highlighting the tensions in health care decision making and grappling with how health practitioners might engage with their ignorance. 


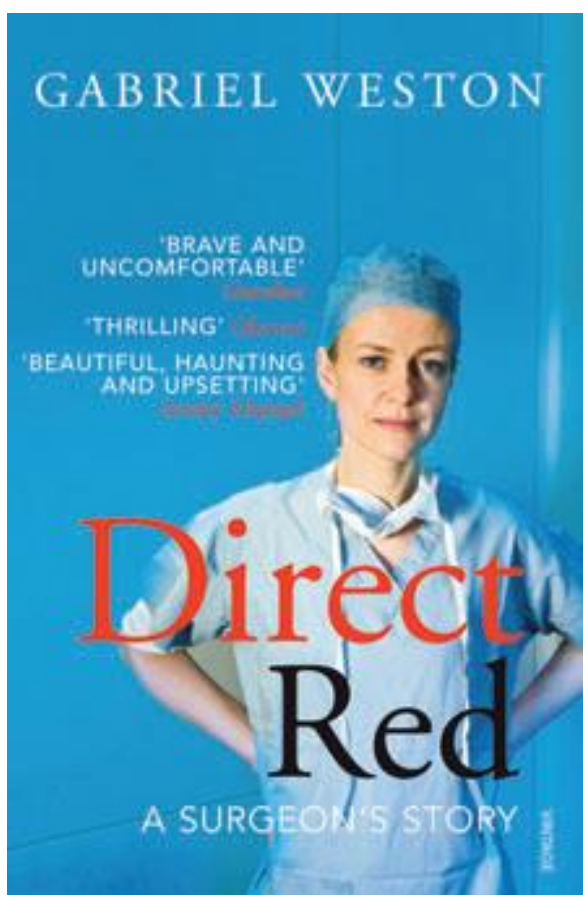

Direct $\operatorname{Red}^{10}$ by Gabriel Weston is my second selection. Her autobiographical account is significant for student introspection because it gives a female surgeon's account of what has traditionally been a male profession. However, like the other two autobiographical accounts, Weston's introspection centres on what it means to be a 'good' practitioner. She is concerned with both technical competence and patient relationships. She explores the work of medical practitioners, highlighting the inevitable tension between emotional detachment and human involvement. She examines the significance of communication, illustrating how health practitioners deny and allow patients' voices. With personal anecdotes, Weston tackles the role that confidence and humility play in health practitioners' practice. For student-readers she offers ways of thinking about and managing the uncertainties and ambiguities of health care practice, highlighting the poignancy even of 'best health care practice.

Even the most righteous surgery can be ugly. Even the most necessary operation, in the best hands, can fail. And in the process of acting in the patient's best surgical interests, we may sometimes make the final moments of their life more terrible than they would ever have been had we left them alone to say their farewells uninterfered with. ${ }^{10}$

My third choice is Mohamed Khadra's Making the cut. ${ }^{11}$ Kha- dra explicitly raises the issues about health, surgery, death and dying that he believes need to be better addressed or better understood both by patients and the health practitioners who look after them. During his time as Professor of Surgery, Khadra required that his students share favourite poems as part of their study programme. He used these poems to help students understand their humanity and that of their patients, to gain insight into the people upon whom they would be performing their surgery. A poem at the beginning of each chapter in this autobiographical account acts as a 
springboard for the issues that Khadra explores. As with the previous two books, Khadra highlights the tension between confidence and humility prerequisite for effective practice. Through poetry, vignettes and discussion, he engages with patients' rights, with death and dying, and offers critical reflection on what doctors are called upon to do. What makes this account unique are the closing chapters. In these final chapters, Khadra passes from being the health practitioner to being a patient, surviving life-threatening throat cancer and consequent surgery. He reflects on illness from a patient's perspective. He engages with the ultimate questions of what it means to be human, discussing mortality, death and the meaning of life. These reflections in themselves are worthy of discussion with student-readers as they filter a patient's experience through the lens of a doctor's understanding, rupturing the divide between patient and physician.

All three autobiographies confront what it means for practitioners (and their patients) to be engaged in 'an imperfect science'. 8 The accounts document the confusion consequent on asking questions the authors are not able to answer. In so doing, they model for novice practitioners the acts of interpretation and contemplation that so significantly complement the technical aspects of treating disease. ${ }^{3}$ 


\section{References}

1. Hudson Jones A. Literature as mirror or lamp? J Clin Ethics 1994;5:340- 341.

2. Charon R. Narrative and medicine. N Engl J Med 2004;350:862-864.

3. Brandon N. Reading more into the medical curriculum: The history of literature and medicine teaching in undergraduate medical education at American and Canadian Medical Schools. Geza HetenyiStudentship for the study of the history of medicine at the University of Ottawa, 2008. Available at http:// www.medicine.uottawa.ca/history ofmedicine/hetenyi/nicholas_ brandon.htm. Accessed on 8 December 2010.

4. Charon R, Trautmann Banks J, Connelly JE, Hunsaker Hawkins A, Montgomery Hunter K, Hudson Jones A, Montello M, Poirer S. Literature and Medicine: Contributions to Clinical Practice. Ann Intern Med 1995;122:599-606.

5. Trilling L. On the teaching of modern literature, in Trilling L. (ed.) Beyond culture: Essays on literature and learning. pp. 3-27. New York: Harcourt Brace Jovanovich; 1965.

6. Kuper A. Literature and medicine: A problem of assessment. Acad Med 2006;81:S128-S137.

7. Calman KC. Literature in the education of the doctor. Lancet 1997;350:1622-1624.

8. Gawande A. Complications. A surgeon's notes on an imperfect science. London: Profile Books; 2002.

9. Dawes RM, Faust D, Meehl PE. Clinical versus actuarial judgment. Science 1989;243:1668-1674.

10. Weston G. Direct red. A surgeon's story. London: Jonathan Cape; 2009.

11. Khadra M. Making the cut: A surgeon's stories of life on the edge. Sydney: Random House; 2007. 